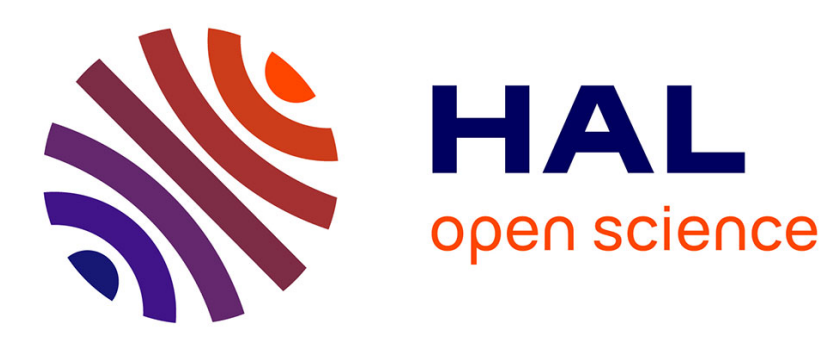

\title{
Dielectrophoretic force-driven thermal convection in annular geometry
}

Harunori N. Yoshikawa, Olivier Crumeyrolle, Innocent Mutabazi

\section{To cite this version:}

Harunori N. Yoshikawa, Olivier Crumeyrolle, Innocent Mutabazi. Dielectrophoretic force-driven thermal convection in annular geometry. Physics of Fluids, 2013, 25, pp.024106. 10.1063/1.4792833 . hal-00904854v2

\section{HAL Id: hal-00904854 \\ https://hal.science/hal-00904854v2}

Submitted on 21 Nov 2013

HAL is a multi-disciplinary open access archive for the deposit and dissemination of scientific research documents, whether they are published or not. The documents may come from teaching and research institutions in France or abroad, or from public or private research centers.
L'archive ouverte pluridisciplinaire HAL, est destinée au dépôt et à la diffusion de documents scientifiques de niveau recherche, publiés ou non, émanant des établissements d'enseignement et de recherche français ou étrangers, des laboratoires publics ou privés. 


\title{
Dielectrophoretic force-driven thermal convection in annular geometry
}

\author{
Harunori N. Yoshikawa, ${ }^{1, \text { a) }}$ Olivier Crumeyrolle, ${ }^{1}$ and Innocent Mutabazi ${ }^{1}$
}

Laboratoire Ondes et Milieux Complexes, UMR 6294 CNRS - Université du Havre

53, rue de Prony - 76058 Le Havre Cedex, France, European Union

(Dated: 21 November 2013)

The thermal convection driven by the dielectrophoretic force is investigated in annular geometry under microgravity conditions. A radial temperature gradient and a radial alternating electric field are imposed on a dielectric fluid that fills the gap of two concentric infinite-length cylinders. The resulting dielectric force is regarded as thermal buoyancy with a radial effective gravity. This electric gravity varies in space and may change its sign depending on the temperature gradient and the cylinder radius ratio. The linear stability problem is solved by a spectral-collocation method. The critical mode is stationary and non-axisymmetric. The critical Rayleigh number and wavenumbers depend sensitively on the electric gravity and the radius ratio. The mechanism behind the instability is examined from an energetic viewpoint. The instability in wide gap annuli is an exact analogue to the gravity-driven thermal instability.

a) Electronic mail: Harunori.Yoshikawa@univ-lehavre.fr 


\section{INTRODUCTION}

The effect of electric fields on the dynamics of insulating or poorly conducting dielectric liquids is concerned with the improvements of flow control and heat transfer. In the latter context, the electric field coupled convection has been investigated by different researchers since Kronig \& Ahsmann's experiment. ${ }^{1}$ Experiments and theoretical analyses were reported for different geometrical configurations of electrodes. ${ }^{2}$ This convection phenomenon has renewed interests in astronautical engineering, where the thermal management of power consuming devices in microgravity is a crucial technological issue. The electric field can provide an artificial gravity and generate thermal convection to enhance the heat transfer in the liquids.

The electrohydrodynamic (EHD) force exerted on a dielectric fluid per unit volume is given by ${ }^{3}$ :

$$
\mathbf{f}_{e}=\rho_{e} \boldsymbol{E}-\frac{1}{2} E^{2} \boldsymbol{\nabla} \epsilon+\boldsymbol{\nabla}\left[\frac{1}{2} \rho\left(\frac{\partial \epsilon}{\partial \rho}\right)_{\theta} E^{2}\right],
$$

where $\boldsymbol{E}$ is the electric field and $E$ is its magnitude. The free electric charge density, the permittivity and the fluid mass density are denoted by $\rho_{e}, \epsilon$ and $\rho$, respectively. The three terms of Eq. (1) represent the electrophoretic, dielectrophoretic (DEP) and electrostrictive forces, respectively. The permittivity $\epsilon$ varies with the temperature. For a small temperature increase $\theta$ from a reference temperature at which $\epsilon=\epsilon_{2}$, it is given by

$$
\epsilon=\epsilon(\theta)=\epsilon_{2}(1-e \theta) .
$$

As we will assume in the present paper, the thermal coefficient of permittivity $e$ is positive (with some exceptional polar liquids, e.g., acetic and butyric acids) and takes typically a value of the order of $10^{-3}-10^{-2} \mathrm{~K}^{-1}$.

In a liquid subjected to a temperature gradient, convection flow can be generated by applying an electric field on it. When a static or low frequency alternating field is applied, the free charge density $\rho_{e}$ appears even in an initially electrically neutral fluid. ${ }^{2}$ This charge accumulation occurs over a time scale of the charge relaxation time $\tau_{e}=\epsilon / \sigma$ through the coupling of the electric field with the spatial gradient of the fluid electrical properties. The electrophoretic force on the built up $\rho_{e}$ is usually the dominant component of the EHD force and can give rise to thermal convection. ${ }^{4,5}$ When the applied electric field has a high 
frequency $f \gg \tau_{e}^{-1}$, the free charge accumulation does not occur. The DEP force is then predominant $^{6}$ and can also develop thermal convection. ${ }^{7-10}$

In the studies on the DEP force-driven thermal convection, the electric gravity is often introduced in order to understand the phenomenon intuitively by analogy with the thermal convection due to the Earth's gravity. The DEP force $\boldsymbol{F}_{D E P}=-E^{2} \boldsymbol{\nabla} \epsilon / 2$ can, in fact, be arranged by invoking Eq. (2) as follows:

$$
\boldsymbol{F}_{D E P}=\nabla\left(\frac{e \theta \epsilon_{2} E^{2}}{2}\right)-\rho \alpha \theta \boldsymbol{g}_{e}
$$

with the electric gravity

$$
\boldsymbol{g}_{e}=\frac{e}{\alpha \rho} \nabla \frac{\epsilon_{2} E^{2}}{2}
$$

where $\alpha$ is the coefficient of thermal expansion. The first term of Eq. (3) is a gradient force that can be lumped with the pressure term. It would not influence convection flows when the fluid is incompressible and has no mobile boundaries. The second term proportional to the temperature is the electric buoyancy force that drives thermal convection. The electric gravity is proportional to the gradient of the electrostatic energy stored per unit volume of the dielectric liquid.

The DEP force-driven thermal convection has been considered in different geometries. In the plane geometry where a liquid fills the gap of two parallel plane electrodes, destabilizing effects of the electric field to the basic conductive state have been recognized both in horizontal and vertical configurations. ${ }^{9,11-13}$ When the terrestrial gravity is neglected, the linear theories predict an instability with the critical value 2129 of the Rayleigh number based on the electric gravity (4) and the critical wavenumber $3.226 / d,,^{9,12}$ where $d$ is the gap size. These critical parameters are different from those of the Rayleigh-Bénard problem (1708 and $3.117 / d$, respectively).

Spherical configuration has also been investigated, having a particular motivation in geoand astrophysics where the understanding of the convection in a spatially varying gravity field in spherical geometry is of primary importance. The DEP force in a liquid layer between two concentric spherical electrodes enables to simulate geo- and astrophysical convection phenomena in laboratory scale systems. Linear stability analyses ${ }^{6,14}$ and numerical simulations ${ }^{15,16}$ with and without rotation of the inner sphere show the generation of convection beyond a critical Rayleigh number. Recently, bifurcation properties of the convection 


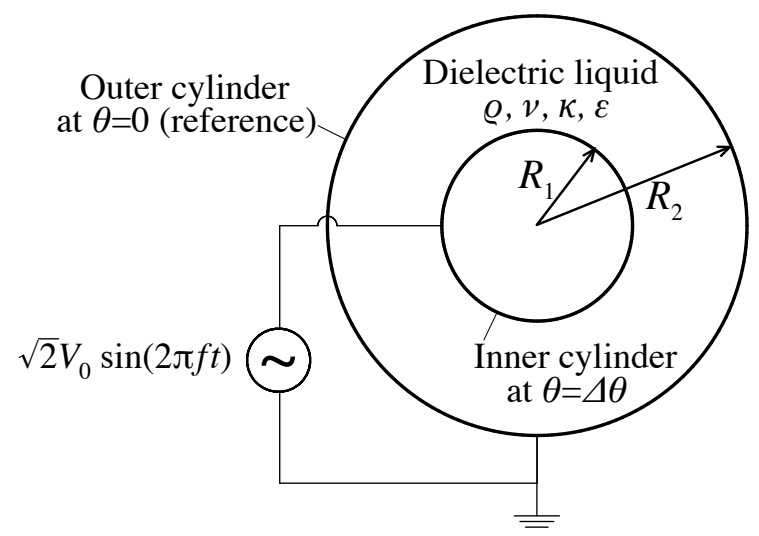

FIG. 1. Schematic illustration of the geometrical configuration in a plane transversal to the annulus axis.

have been examined in detail from the viewpoint of the dynamical systems theory. ${ }^{16}$ Experiments performed in the Fluid Science Laboratory on the board of the International Space Station show a favorable agreement with the theoretical predictions. ${ }^{17,18}$

In the literature, there are some studies in annular geometry. This geometry is concerned with applications in heat management for, e.g., motor systems. The linear stability of the Taylor-Couette flows with a radial DEP force field was considered. ${ }^{19}$ The research is also motivated by the geo- and astrophysical applications, regarding the thermal convection in this geometry as a lower level simulation system. ${ }^{7}$ Chandra \& Smylie ${ }^{7}$ realized an experiment on the ground with stationary vertical cylinders, choosing parameters for which the Earth's gravity is negligible (i.e., small Grashof numbers). They performed a linear stability analysis to axisymmetric disturbances for their experimental parameters and found a reasonable agreement with the experiment. In their analysis, the influence of the temperature gradient on the electric field was neglected. This thermo-electric coupling has been taken into account in the linear stability analysis by Takashima ${ }^{8}$ for narrow gap situations.

In the present work which is an extension of a recently published work,${ }^{20}$ we consider the DEP convection in the annular geometry under microgravity conditions (Fig. 1), aiming to examine the stability over a wide range of control parameters (the gap size and the thermal variation of $\epsilon$ ) and to unveil the mechanism behind the instability. The cylindrical electrodes are assumed as perfect conductors and of infinite length in order to examine the instability in its simplest configuration. In the next section, we present the governing equations and discuss the basic conductive state and the basic electric gravity. In Sec. III, the linear 
stability problem is formulated and its symmetry properties are discussed. Determined critical parameters and eigenmodes are presented in Sec. IV. Section V is devoted to a discussion on the instability mechanism from an energetic viewpoint. In Sec. VI, we compare the results with the previous works and give our conclusions.

\section{GOVERNING EQUATIONS AND BASIC STATE}

\section{A. Governing equations}

Equations governing dielectric fluid motion in a high frequency alternating electric field are presented in Turnbull's paper ${ }^{11}$ in their general forms. All the equations are timeaveraged over a period of the electric field with replacing the imposed electric potential $\sqrt{2} V_{0} \sin (2 \pi f t)$ by its effective value $V_{0}$. This time-averaged description is valid when the frequency is high compared to the inverse of the viscous time scale $d^{2} / \nu$. It predicted successfully the onset of the DEP thermal convection. ${ }^{21}$ The equations include $\rho, \nu$ and the thermal diffusion coefficient $\kappa$ which are functions of temperature $\theta$. We assume that these parameters are constant, adopting the electrohydrodynamic Boussinesq approximation ${ }^{11}$, in which only the permittivity varies with the temperature. This approximation is valid as long as the imposed temperature difference is not large. ${ }^{11}$ We have then the equations of continuity, motion and heat conduction and the Gauss's law of electricity as follows:

$$
\begin{aligned}
\boldsymbol{\nabla} \cdot \boldsymbol{u} & =0 \\
\frac{\partial \boldsymbol{u}}{\partial t}+\boldsymbol{u} \cdot \boldsymbol{\nabla} \boldsymbol{u} & =-\nabla \pi+\nu \triangle \boldsymbol{u}-\alpha \theta \boldsymbol{g}_{e}, \\
\frac{\partial \theta}{\partial t}+\boldsymbol{u} \cdot \boldsymbol{\nabla} \theta & =\kappa \triangle \theta, \\
\nabla \cdot(\epsilon \boldsymbol{E}) & =0 \quad \text { with } \quad \boldsymbol{E}=-\boldsymbol{\nabla} \phi
\end{aligned}
$$

where $\boldsymbol{u}$ is the velocity field and $\phi$ is the electric potential. The generalized pressure $\pi$ is related to the hydrodynamic pressure $p$ as

$$
\pi=\frac{p}{\rho}-\frac{e \theta \epsilon_{2} E^{2}}{2 \rho}-\frac{1}{2}\left(\frac{\partial \epsilon}{\partial \rho}\right)_{\theta} E^{2} .
$$


Viscous energy dissipation and Joule heating have been neglected in Eq. (7). The former is small in convection flows of incompressible fluid. ${ }^{22}$ The Joule heating relative to the heat conduction is estimated as $6.29 V_{0}^{2} f \epsilon \tan \delta / \lambda \Delta \theta_{\text {ref }}(\delta$ : the dielectric loss angle, $\lambda$ : the thermal conductivity, $\Delta \theta_{\text {ref: }}$ temperature difference),${ }^{6}$ giving a tiny value in typical conditions, e.g., 0.007 for silicone oils when $f=60 \mathrm{~Hz}, V_{0}=10 \mathrm{kV}$ and $\Delta \theta_{\text {ref }}=1 \mathrm{~K}$.

In addition to the governing equations (5)-(8), we may refer to the vorticity equation which is derived from Eq. (6):

$$
\frac{\partial \boldsymbol{\zeta}}{\partial t}+\boldsymbol{u} \cdot \nabla \boldsymbol{\zeta}=\boldsymbol{\zeta} \cdot \nabla \boldsymbol{u}+\nu \triangle \boldsymbol{\zeta}-\alpha \boldsymbol{\nabla} \theta \times \boldsymbol{g}_{e}
$$

where $\boldsymbol{\zeta}$ is the vorticity: $\boldsymbol{\zeta}=\boldsymbol{\nabla} \times \boldsymbol{u}$. The last term in the right-hand-side is the source of vorticity generation; the electric gravity that is not parallel to the temperature gradient generates vorticity to develop convection.

We consider the fluid filling the gap of two concentric cylinders as illustrated in Fig. 1, where $R_{1}$ and $R_{2}$ are the radii of the inner and outer cylinders, respectively. These cylinders are kept at uniform temperatures and electric potentials, with an imposed temperature difference $\Delta \theta$ and a high frequency electric potential over the gap. In the time-averaged description, the boundary conditions at the cylinders are

$$
\left\{\begin{array}{l}
\boldsymbol{u}=0, \theta=\Delta \theta, \phi=V_{0} \text { at } r=R_{1}, \\
\boldsymbol{u}=0, \quad \theta=0, \quad \phi=0 \quad \text { at } r=R_{2} .
\end{array}\right.
$$

\section{B. Basic conductive state}

When $|\Delta \theta|$ and $V_{0}$ are small, the state of the system will be steady, axisymmetric and axially invariant. The temperature and the electric potential will depend only on the radial coordinate $r: \theta=\bar{\theta}(r), \phi=\bar{\phi}(r)$. The temperature gradient and the electric gravity are then collinear with each other so that the source term in the vorticity equation (10) is null; there is no flow: $\overline{\boldsymbol{u}}=0$.

For this basic state, Eqs. (7) and (8) read in the cylindrical coordinates $(r, \varphi, z)$ :

$$
\frac{1}{r} \frac{d}{d r}\left(r \frac{d \bar{\theta}}{d r}\right)=0 \quad \text { and } \quad \frac{1}{r} \frac{d}{d r}\left(r \bar{\epsilon} \frac{d \bar{\phi}}{d r}\right)=0
$$



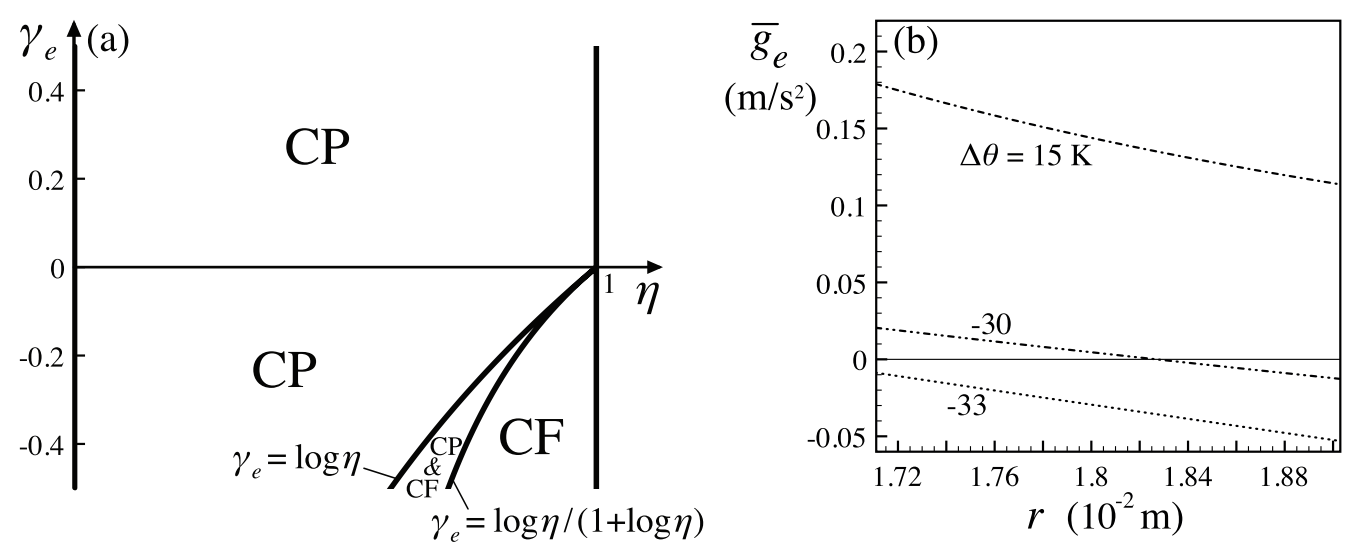

FIG. 2. Basic electric gravity $\boldsymbol{g}_{e}=-\bar{g}_{e} \mathbf{e}_{r}$. (a) Its direction as function of the dimensionless temperature $\gamma_{e}$ and the radius ratio $\eta$. CP and CF mean centripetal and centrifugal, respectively. (b) Some profiles of $\bar{g}_{e}$ calculated for the electric tension $V_{0}=1.08 \times 10^{4} \mathrm{~V}$. The cylinder radii and liquid properties are taken from the Chandra \& Smylie's experiment ${ }^{7}\left(R_{1}=1.711 \times 10^{-2} \mathrm{~m}\right.$, $R_{2}=1.903 \times 10^{-2} \mathrm{~m}, \rho=937.7 \mathrm{~kg} \mathrm{~m}^{-3}, \alpha=1.08 \times 10^{-3} \mathrm{~K}^{-1}$ and $\left.e=3.7 \times 10^{-3} \mathrm{~K}^{-1}\right)$.

where $\bar{\epsilon}$ is the permittivity at the local temperature $\bar{\theta}(r)$. Solving these equations with the boundary conditions (11), we have

$$
\bar{\theta}=\frac{\Delta \theta \log \left(r / R_{2}\right)}{\log \eta}, \quad \bar{\phi}=\frac{V_{0} \log \left(1-\gamma_{e} \bar{\theta} / \Delta \theta\right)}{\log \left(1-\gamma_{e}\right)}
$$

where $\eta=R_{1} / R_{2}$ is the radius ratio and $\gamma_{e}=e \Delta \theta$ is the dimensionless temperature difference that represents the thermal variation of $\epsilon$. The basic electric field $\bar{E} \mathbf{e}_{r}$ is computed from $\bar{E}=-d \bar{\phi} / d r:$

$$
\bar{E}=\frac{\gamma_{e} V_{0}}{r\left(1-\gamma_{e} \bar{\theta} / \Delta \theta\right) \log \left(1-\gamma_{e}\right) \log \eta} .
$$

The resulting electric gravity (4) from this basic electric field is radial and is given by $\boldsymbol{g}_{e}=-\bar{g}_{e} \mathbf{e}_{r}$ with

$$
\bar{g}_{e}=\frac{e \epsilon_{2} V_{0}^{2}}{\alpha \rho(\log \eta)^{2} r^{3}} F, \quad \text { where } \quad F=\frac{\gamma_{e}^{2}\left[1-\gamma_{e}(\bar{\theta} / \Delta \theta+1 / \log \eta)\right]}{\left[\log \left(1-\gamma_{e}\right)\right]^{2}\left(1-\gamma_{e} \bar{\theta} / \Delta \theta\right)^{3}}
$$

This electric gravity has two contributing factors. The first is the electric field inhomogeneity due to the geometry curvature. It brings about the behavior inversely proportional to $r^{3}$. The second is the inhomogeneity due to the thermo-electric coupling represented by the factor $F$. This coupling makes the electric gravity behavior different for different $\gamma_{e}$ (Fig. 2a). When 
the heating is outward $\left(\gamma_{e}>0\right)$ or inward by a small amount $\left(\log \eta<\gamma_{e}<0\right)$, the electric gravity is centripetal everywhere in the gap. For larger inward heating $(\log \eta /(1+\log \eta)<$ $\left.\gamma_{e}<\log \eta\right)$ the gravity is centripetal and centrifugal, respectively, in the inner and outer sublayers separated at a radial position $r=R_{2} \exp \left(\log \eta / \gamma_{e}-1\right)$. Further increase in inward heating $\left(\gamma_{e}<\log \eta /(1+\log \eta)\right)$ makes the gravity centrifugal throughout the gap.

In the limit of $\gamma_{e} \rightarrow 0$, there is no thermo-electro coupling. The electric gravity is centripetal independent of the heating direction and exhibits the purely inverse cubic behavior. In another limit where the gap $d\left(=R_{2}-R_{1}\right)$ is small: $d / R_{1}=\eta^{-1}-1 \ll\left|\gamma_{e}\right|$, the electric gravity (15) recovers that between two parallel plane electrodes ${ }^{9}$ :

$$
\bar{g}_{e}=\frac{e \epsilon_{2} V_{0}^{2} \gamma_{e}^{3}}{\rho \alpha d^{3}\left[\log \left(1-\gamma_{e}\right)\right]^{2}\left(1-\gamma_{e}+\gamma_{e} x\right)^{3}},
$$

where $x$ is the dimensionless distance from the inner cylinder: $x=\left(r-R_{1}\right) / d$. The gravity is directed from the low to high temperature electrodes.

Some profiles of $\bar{g}_{e}$ are shown in Fig. $2 \mathrm{~b}$ for different values of $\Delta \theta$. The cylinder radii and the fluid properties are taken from the experiment of Chandra \& Smylie. ${ }^{7}$ With decreasing $\Delta \theta$, the electric gravity varies from centripetal to centrifugal. The profiles are not symmetric with respect to the change of heating direction, since the electric gravity (15) is primarily related to the curvature of the cylindrical geometry and not to the thermal variation of permittivity except for extremely narrow gaps.

\section{LINEAR STABILITY PROBLEM}

Using scales $d$ of length, $d^{2} / \kappa$ of time, $g_{0}$ of electric gravity and $\Delta \theta$ of temperature, we nondimensionalize the governing equations (5)-(8). Dimensionless parameters involved are the radius ratio $\eta$, the dimensionless temperature difference $\gamma_{e}$, the Prandtl number $\operatorname{Pr}=\nu / \kappa$ and the electric Rayleigh number $L$ :

$$
L=\frac{\alpha \Delta \theta g_{0} d^{3}}{\kappa \nu}
$$

The latter number is the Rayleigh number based on the electric gravity. We estimate the representative electric gravity $g_{0}$ at the middle of the gap $r=r_{0}$, where $r_{0}=(1+\eta) / 2(1-\eta)$. 
The electric Rayleigh number (17) is positive when the gravity at this reference point is in the same direction as the basic temperature gradient; otherwise, it takes a negative value.

The nondimensionalized governing equations are linearized about the basic state. The perturbations are developed into normal modes of complex growth rate $s$, azimuthal mode number $n$ and axial wavenumber $k$ : $(\tilde{u}, \tilde{v}, \tilde{w}, \tilde{\pi}, \tilde{\theta}, \tilde{\phi}) e^{s t+i n \varphi+i k z}$, where $\tilde{u}, \tilde{v}$ and $\tilde{w}$ are the complex amplitudes of the radial, azimuthal and axial perturbation velocity components, respectively. Those of the perturbation pressure, temperature and electric potential are denoted by $\tilde{\pi}, \tilde{\theta}$ and $\tilde{\phi}$. The resulting equations are:

$$
\begin{aligned}
& \frac{1}{r}(r \tilde{u})^{\prime}+\frac{i n}{r} \tilde{v}+i k \tilde{w}=0, \\
& \triangle \tilde{u}-\frac{\tilde{u}}{r^{2}}-\frac{2 i n}{r^{2}} \tilde{v}-\tilde{\pi}^{\prime}+L\left(\bar{g}_{e} \tilde{\theta}-\tilde{g}_{r} \bar{\theta}\right)=\operatorname{Pr}^{-1} s \tilde{u}, \\
& \triangle \tilde{v}-\frac{\tilde{v}}{r^{2}}+\frac{2 i n}{r^{2}} \tilde{u}-\frac{i n}{r} \tilde{\pi}-L \bar{\theta} \tilde{g}_{\varphi}=\operatorname{Pr}^{-1} s \tilde{v}, \\
& \triangle \tilde{w}-i k \tilde{\pi}-L \bar{\theta} \tilde{g}_{z}=\operatorname{Pr}^{-1} s \tilde{w}, \\
& \triangle \tilde{\theta}-\tilde{u} \bar{\theta}^{\prime}=\operatorname{Pr}^{-1} s \tilde{\theta}, \\
& \bar{\epsilon} \triangle \tilde{\phi}+\bar{\epsilon}^{\prime} \tilde{\phi}^{\prime}-\gamma_{e} \bar{\phi}^{\prime} \tilde{\theta}^{\prime}-\gamma_{e} \tilde{\theta}\left(\bar{\phi}^{\prime \prime}+\frac{\bar{\phi}^{\prime}}{r}\right)=0,
\end{aligned}
$$

where the primes signify a derivation with respect to $r$. The Laplacian operator $\triangle$ and the components of perturbation electric gravity $\left(\tilde{g}_{r}, \tilde{g}_{\varphi}, \tilde{g}_{z}\right)$ have been introduced:

$$
\begin{gathered}
\triangle=\frac{d^{2}}{d r^{2}}+\frac{1}{r} \frac{d}{d r}-\left(\frac{n^{2}}{r^{2}}+k^{2}\right), \\
\tilde{g}_{r}=C\left(\eta, \gamma_{e}\right)\left(\bar{E}^{\prime} \tilde{\phi}^{\prime}+\bar{E} \tilde{\phi}^{\prime \prime}\right), \quad \tilde{g}_{\varphi}=\frac{i n}{r} C\left(\eta, \gamma_{e}\right) \bar{E} \tilde{\phi}^{\prime}, \quad \tilde{g}_{z}=i k C\left(\eta, \gamma_{e}\right) \bar{E} \tilde{\phi}^{\prime} .
\end{gathered}
$$

The coefficient $C=C\left(\eta, \gamma_{e}\right)$ is given by:

$$
C=-\frac{\left[\log \left(1-\gamma_{e}\right)\right]^{2} r_{0}^{3}\left(\log \eta-\gamma_{e} \log \left[(1-\eta) r_{0}\right]\right)^{3}}{\gamma_{e}^{2}\left[\log \eta-\gamma_{e}\left(\log \left[(1-\eta) r_{0}\right]+1\right)\right]}
$$

The boundary conditions for perturbations are derived from (11) and read:

$$
\tilde{u}=\tilde{v}=\tilde{w}=\tilde{u}^{\prime}=\tilde{\theta}=\tilde{\phi}=0 .
$$




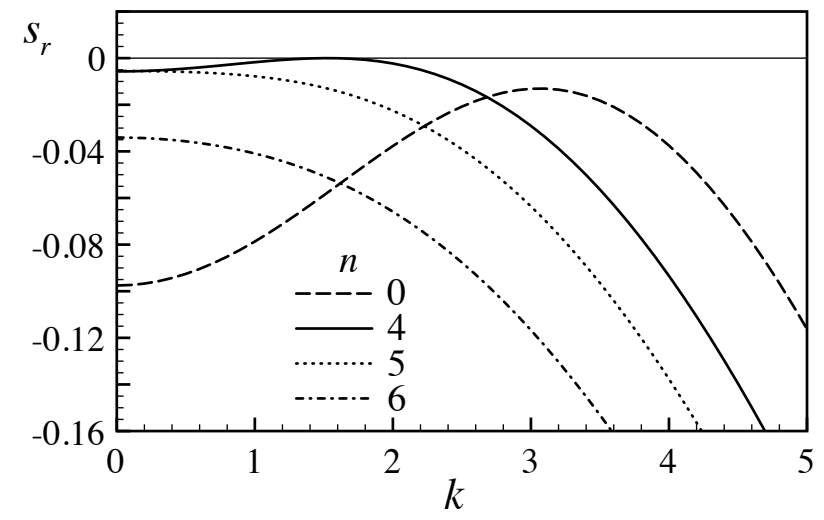

FIG. 3. Dispersion relation for different azimuthal mode number $n$. $\left(\operatorname{Pr}=100, \eta=0.5, \gamma_{e}=0.01\right.$, $L=1498)$

The Gauss's law (23) expresses the feedback of the electric field to temperature disturbances through the thermo-electro coupling. The resulting electric field is transmitted into the dynamical equations (19)-(21) through the perturbation electric gravity. When the imposed temperature difference $\gamma_{e}$ is negligibly small, Eq. (23) admits only the trivial solution under the boundary condition (27). There is then no feedback effect: no perturbation electric gravity is generated.

Equations (18)-(23) and the boundary conditions (27) form an eigenvalue problem which determines the stability of the basic state against toroidal $(n=0, k \neq 0)$, helical $(n \neq 0$, $k \neq 0)$ or columnal $(n \neq 0, k=0)$ disturbances. When the instability occurs, these disturbances develop into convection rolls.

The problem has a geometrical invariance associated with the mirror symmetry with respect to the meridian plane $\varphi=0$; Eqs. (18)-(23) and (27) are invariant by the operation $(n, \tilde{v}) \rightarrow(-n,-\tilde{v})$. Once the eigenvalue $s$ and the eigenfunctions $(\tilde{u}, \tilde{v}, \tilde{w}, \tilde{\pi}, \tilde{\theta}, \tilde{\phi})$ are known for a spatial mode $(n, k)$, those for the mode $(-n, k)$ are then given by $(s, \tilde{u},-\tilde{v}, \tilde{w}, \tilde{\pi}, \tilde{\theta}, \tilde{\phi})$. The stability conditions of both modes are identical to each other. Similar discussion can be made about another invariance to the operation $(k, \tilde{w}) \rightarrow(-k,-\tilde{w})$ that reflects the mirror symmetry with respect to the transversal plane $z=0$. The stability does not, therefore, depend on the signs of $n$ and $k$. We solve the eigenvalue problem for positive $n$ and $k$ by a spectrum collocation method, the details of which are given in appendix A. 


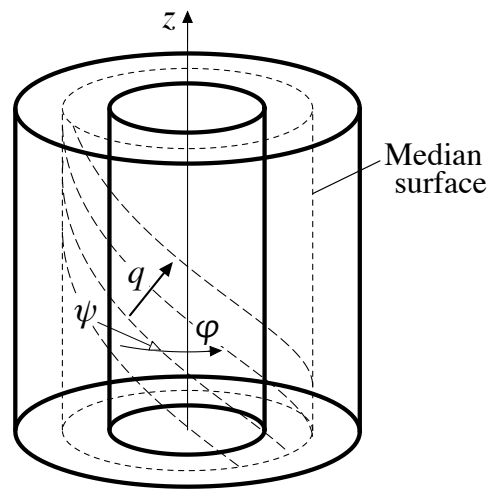

FIG. 4. Definitions of the wavenumber $q$ and the inclination angle $\psi$ of convection rolls.

\section{RESULTS}

\section{A. Dispersion relation \& Marginal curves}

Eigenvalues $s=s_{\mathrm{r}}+\mathrm{i} s_{\mathrm{i}}$ with the largest real part are computed for a given parameter set $\left(\eta, \operatorname{Pr}, \gamma_{e}, L, n\right)$ with varying the axial wavenumber $k$. Obtained dispersion curves $s_{\mathrm{r}}=$ $s_{\mathrm{r}}(k)$ have their maxima at a finite $k$ for small $n$ and at $k=0$ for $n$ larger than a certain value $m$ (Fig. 3), where $m$ is the maximum number of convection roll pairs of the gap size: $m=[\pi(1+\eta) / 2(1-\eta)]$. The square brackets indicate the integer part of the quantity enclosed. The eigenmodes are stationary $\left(s_{\mathrm{i}}=0\right)$, implying that the principle of the exchange of stabilities holds as in the Rayleigh-Bénard problem. ${ }^{11,22}$ Turnbull gave a proof for the plane geometry DEP convection with free boundary conditions. ${ }^{11}$

An eigenmode is associated with convection rolls whose basic geometrical characteristics are specified by the wavenumber $q$ measured along the direction transversal to the rolls and by the inclination angle $\psi$ to the azimuthal direction. Both are estimated on the median cylindrical surface $r=r_{0}$ (Fig. 4) and given by

$$
q=\sqrt{\frac{4 n^{2}(1-\eta)^{2}}{(1+\eta)^{2}}+k^{2}}, \quad \psi=\tan ^{-1} \frac{2 n(1-\eta)}{k(1+\eta)}
$$

Figure 5 shows marginal curves as function of $q$ for different azimuthal mode number $n$. Some curves come to an end because $q$ is lower bounded by its value at $k=0$. For small $\eta$, the minima of different marginal curves are distinct (Fig. 5a), while for large $\eta$ they degenerate with each other (Fig. 5b). This mode degeneration is due to the rotational symmetry with 

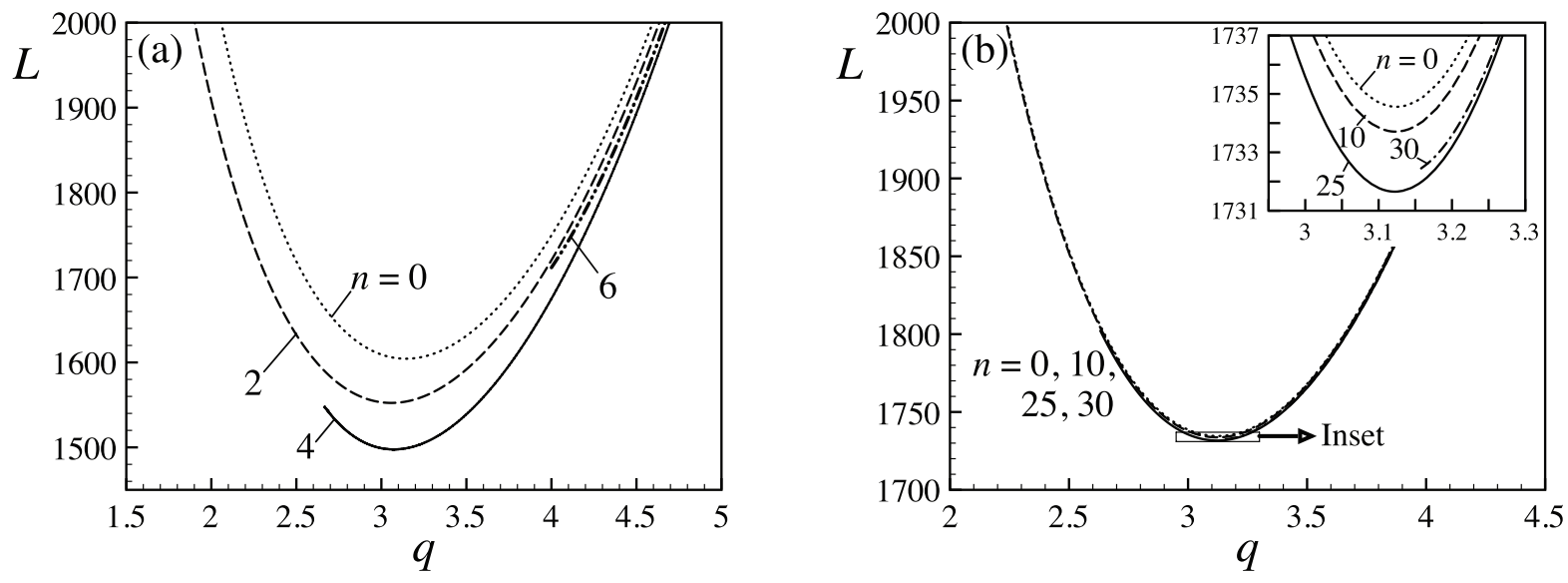

FIG. 5. Marginal curves for different azimuthal mode number $n$ at small and large radius ratios $\eta$ : (a) $\eta=0.5$ and (b) $\eta=0.9$. The dimensionless temperature $\gamma_{e}$ is fixed at 0.01 .

respect to wall normals, which is recovered locally as $\eta$ increases.

\section{B. Critical Rayleigh number}

Increase of the magnitude of the control parameter $L$ leads to an instability, but only either in the case of outward heating $\left(\gamma_{e}>0\right)$ or in the case of inward heating with narrow gaps $\left(\gamma_{e}<\log \eta /(1+\log \eta)\right)$. In these cases, the basic electric gravity is centripetal and centrifugal throughout the gap, respectively, and in the same direction as the imposed temperature gradient. The electric Rayleigh number $L$ should hence be positive for the instability. The critical parameters are independent of the Prandtl number. ${ }^{20}$ This is consistent with the observation that the instability is stationary: as Pr appears only in the coefficients of $s$ in Eqs. (18)-(23), its value cannot influence the marginal stability conditions of the stationary modes.

Figure 6 shows the critical electric Rayleigh number $L_{c}$ for different imposed temperature $\gamma_{e}$. When the heating is outward $\left(\gamma_{e}>0\right), L_{c}$ increases monotonically. The non-smooth behavior is related to the discontinuous changes of the critical azimuthal mode number $n_{c}$. For small $\eta(\lesssim 0.6), L_{c}$ is independent of $\gamma_{e}$ and behaves similarly to that in the case of $\gamma_{e} \approx 0$. This suggests that for small $\eta$ the instability is driven by the same mechanism as in the gravity-driven ordinary thermal convection, as no perturbation electric gravity is generated for $\gamma_{e}=0$. At large $\eta, L_{c}$ recovers the value of the Rayleigh-Bénard problem (1708). In the narrow gap limit: $\eta \rightarrow 1$ it converges towards the larger value (2129) predicted for the 


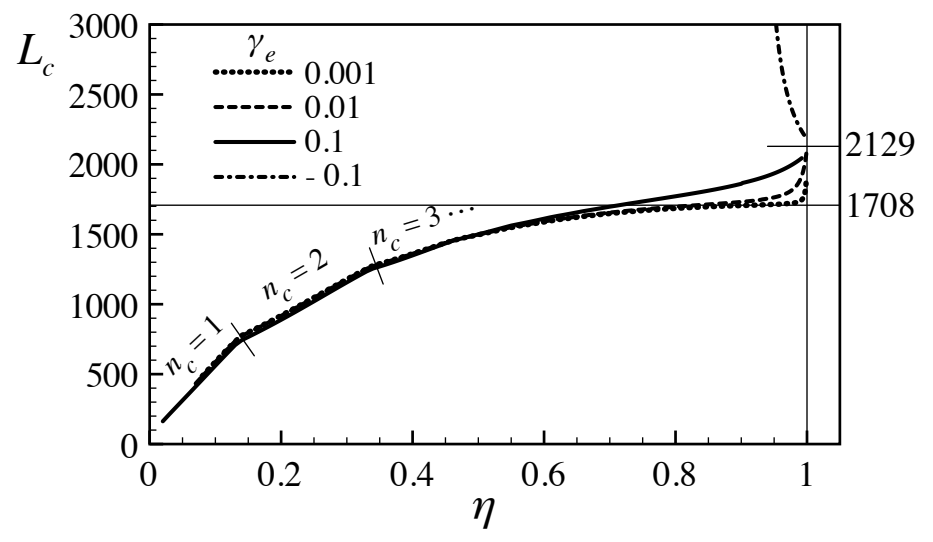

FIG. 6. Critical electric Rayleigh number $L_{c}$ as function of the radius ratio $\eta$ for different dimensionless temperatures $\gamma_{e}$. The horizontal lines show the critical Rayleigh number of the RayleighBénard instability (1708) and $L_{c}$ of the DEP convection in plane geometry (2129). ${ }^{9}$

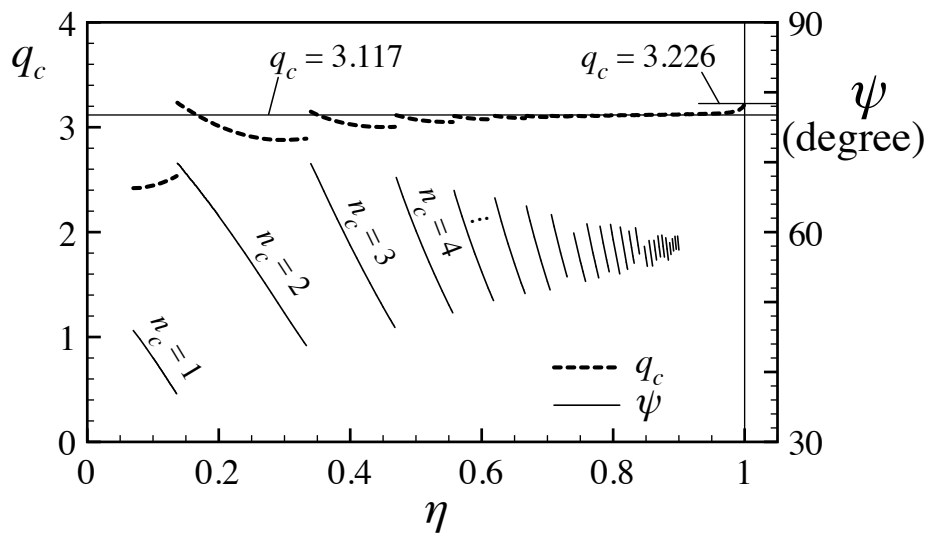

FIG. 7. Critical wavenumber $q_{c}$ and inclination angle $\psi$ for an outward heating $\left(\gamma_{e}=0.01\right)$.

DEP convection in plane geometry. ${ }^{9,12}$ This final increase is delayed to larger $\eta$ for small $\gamma_{e}$.

When the heating is inward $\left(\gamma_{e}<0\right), L_{c}$ also converges to 2129 in the narrow gap limit. For $\eta<1$, it becomes larger and diverges as $\eta$ approaches the boundary between the zones labeled CF and CP \& CF in Fig. 2a. This behavior different from the outward heating case suggests that there will be important differences in the instability mechanism.

\section{Wavenumber selection}

The critical mode number $n_{c}$ increases with $\eta$, being roughly equal to the maximum number of convection roll pairs $m .{ }^{23,24}$ The wavenumber $q_{c}$ for outward heating is nearly 


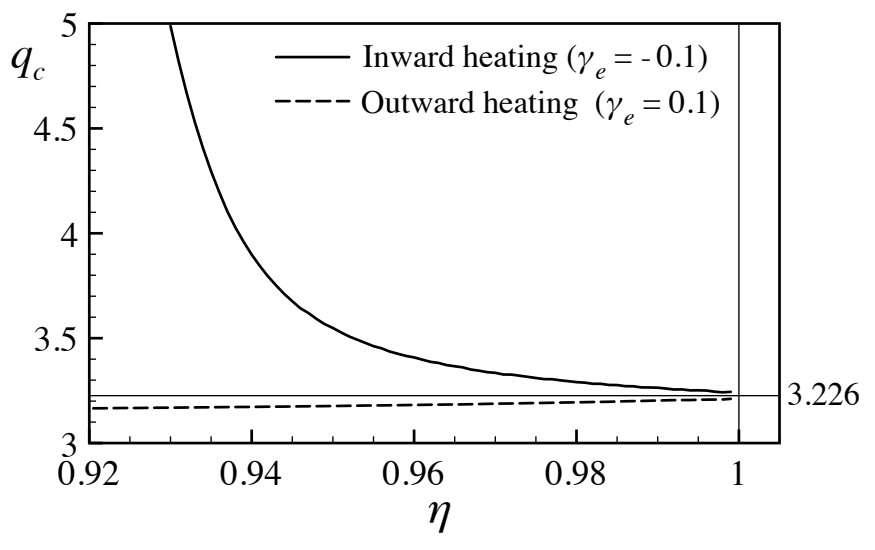

FIG. 8. Critical wavenumber $q_{c}$ for inward and outward heatings.

constant around 3 and identical to the value of the Rayleigh-Bénard instability (3.117) at large $\eta$ (Fig. 7). In the narrow gap limit, $q$ recovers its value predicted by the plane geometry problem (3.226). ${ }^{9,12}$ The angle $\psi$ decreases as long as the mode belongs to an azimuthal mode number $n$. It does not reach neither 0 nor $90^{\circ}$; the convection rolls are made of helices. When $\eta$ is large, the variation of the angle $\psi$ is small. For $\eta$ close to unity, the angle becomes undetermined due to the mode degeneracy. The similar behavior of $q_{c}$ and $\psi$ is found for other positive $\gamma_{e}$, while the final increase of $q_{c}$ towards 3.226 is delayed to larger $\eta$ for small $\gamma_{e}$.

For inward heating, the angle $\psi$ is undetermined, as the instability occurs only for $\eta$ close to unity. As to the wavenumber $q$, it increases rapidly from 3.226 as $\eta$ decreases (Fig. 8). The asymmetry with respect to the heating direction becomes significant as soon as $\eta$ is lowered from unity.

\section{Eigenmodes}

Eigenfunctions exhibit a typical thermal convection pattern as seen in Fig. 9 where the perturbation velocity, temperature, electric gravity and electric potential fields are shown for an outward heating in transversal and meridian sections. Fluid flows from the inner to the outer walls inside the regions of positive perturbation temperature, as the basic electric gravity is centripetal. It flows inversely inside the regions of negative temperature perturbation. When $\eta$ is small, the perturbation fields concentrate in an inner region. In particular, the perturbation electric gravity is almost null except the vicinity of the inner 


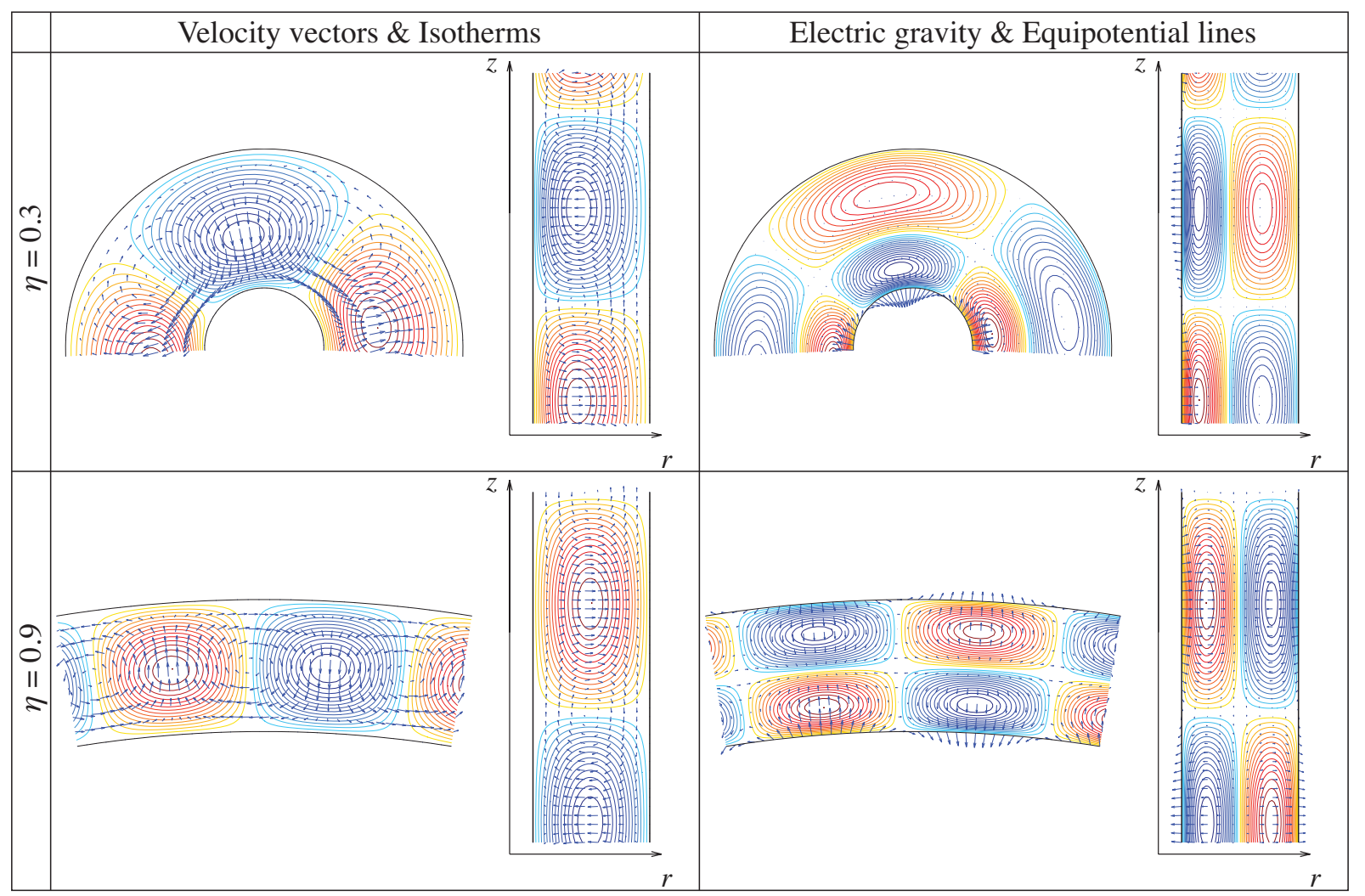

FIG. 9. Critical eigenmodes for small and large radius ratios $\eta$. For $\eta=0.3, n=2, k=1.91$ and $L=1177$. For $\eta=0.9, n=25, k=1.68$ and $L=1732$. The heating is outward with the dimensionless temperature $\gamma_{e}=0.01$ for both cases.

wall. This suggests that the thermo-electro feedback would not have significant effects. In contrast, for large $\eta$, fields are more uniformly distributed over the gap. The perturbation gravity extends over the whole gap, indicating its non-negligible role in the instability.

For inward heating, eigenfunctions show that the perturbation flow goes from the outer (inner) to inner (outer) walls inside positive (negative) perturbation temperature zones, since the basic electric gravity is centrifugal. It also shows that the perturbation electric gravity also extends over the gap; the thermo-electric feedback would be important as in the case of outward heating with large $\eta$.

\section{ENERGY ANALYSIS}

The kinetic energy of convection is given by $\mathcal{K}=\left(|\tilde{u}|^{2}+|\tilde{v}|^{2}+|\tilde{w}|^{2}\right) / 2$ per unit volume, where the energy has been averaged over the spatial periods along $\varphi$ and $z$. An evolution 


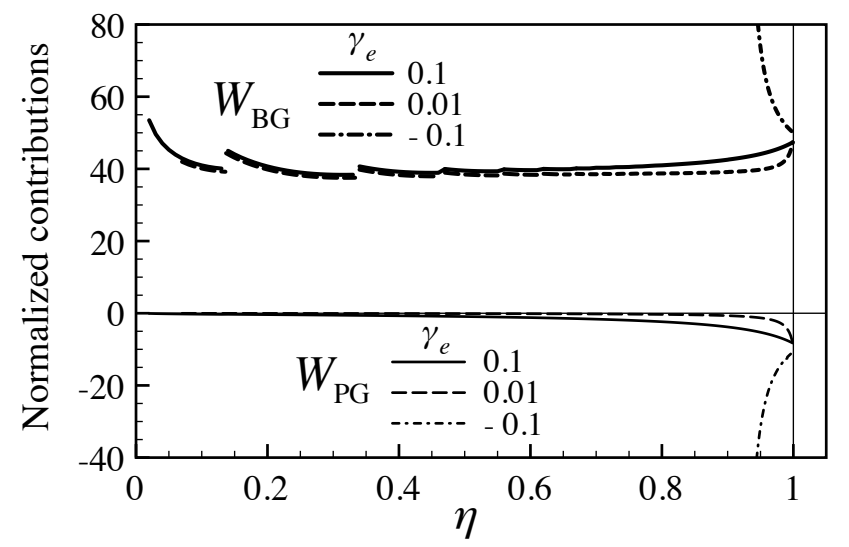

FIG. 10. Normalized different contributions to the rate of change of the convection flow kinetic energy (Eq. 29) at critical conditions.

equation for the total kinetic energy $K=\int r \mathcal{K} d r$ is derived from Eqs. (19)-(21). Multiplying Eqs. (19)-(21) by $\tilde{u}^{*}, \tilde{v}^{*}$ and $\tilde{w}^{*}$, respectively (the asterisks mean complex conjugate), summing them up and integrating over the gap with a weight $r$, we have

$$
2 s K=W_{\mathrm{BG}}+W_{\mathrm{PG}}-D_{\mathrm{v}},
$$

where $W_{\mathrm{BG}}$ and $W_{\mathrm{PG}}$ are the works done by the basic and perturbation electric gravities, respectively, and the last term $D_{\mathrm{v}}$ represents the rate of the viscous dissipation:

$$
\begin{aligned}
W_{\mathrm{BG}} & =-\operatorname{Pr} L \int r \bar{g}_{e}\left(\tilde{\theta} \tilde{u}^{*}+\text { c.c. }\right) d r, \\
W_{\mathrm{PG}} & =\gamma_{e}^{-1} \operatorname{Pr} L \int r\left(1-\gamma_{e} \bar{\theta}\right)\left[\left(\tilde{u}^{*} \tilde{g}_{r}+\tilde{v}^{*} \tilde{g}_{\varphi}+\tilde{w}^{*} \tilde{g}_{z}\right)+c . c .\right] d r, \\
D_{\mathrm{v}} & =\operatorname{Pr} \int r \Phi_{\mathrm{v}}(\tilde{u}, \tilde{v}, \tilde{w}) d r .
\end{aligned}
$$

In these equations, c.c. indicates the complex conjugate of its preceding term and $\Phi_{\mathrm{v}}$ is the viscous dissipation function in the cylindrical coordinates:

$$
\begin{gathered}
\Phi_{\mathrm{v}}=\left|\tilde{u}^{\prime}\right|^{2}+\left|\frac{i n \tilde{u}}{r}-\frac{\tilde{v}}{r}\right|^{2}+k^{2}|\tilde{u}|^{2}+\left|\tilde{v}^{\prime}\right|^{2}+\left|\frac{i n \tilde{v}}{r}+\frac{\tilde{u}}{r}\right|^{2} \\
+k^{2}|\tilde{v}|^{2}+\left|\tilde{w}^{\prime}\right|^{2}+\left|\frac{i n \tilde{w}}{r}\right|^{2}+k^{2}|\tilde{w}|^{2}
\end{gathered}
$$


Figure 10 shows the variation with $\eta$ of the works $W_{\mathrm{BG}}$ and $W_{\mathrm{PG}}$ normalized by $2 K$ for the critical modes. The basic gravity contribution $W_{\mathrm{BG}}$ is predominant and supplies energy to perturbation flow. Two different dynamical regimes can be distinguished according to the importance of $W_{\mathrm{PG}}$ : the first regime where it is almost null; the second regime where $W_{\mathrm{PG}}$ takes a non-negligible negative value.

For outward heating $\left(\gamma_{e}>0\right)$, the first regime is found at small $\eta(\lesssim 0.6)$, where smallness of $W_{\mathrm{PG}}$ can be inferred from eigenfunctions (Fig. 9): the perturbation gravity is in fact localized in the vicinity of the inner cylinder. As the flow is weak in the wall regions, the perturbation gravity does not perform significant work. Negligible $W_{\mathrm{PG}}$ confirms that the feedback through the thermo-electro coupling can be omitted in the stability analysis in this regime. Convection can hence be regarded as an exact analogue to the convection by the ordinary thermal instability due to a central gravity field. The second regime is found at large $\eta$. As seen in Fig. 9, the perturbation gravity field extends inside the gap and can put (draw) energy into (from) perturbation flow locally. The negative contribution of $W_{\mathrm{PG}}$ means that the perturbation gravity has a stabilizing effect in total, being consistent with the increase of $L_{c}$ as $\eta \rightarrow 1$ (Fig. 6). Stabilization by $W_{\mathrm{PG}}$ explains why $L_{c}$ of the DEP convection in plane geometry ${ }^{9,12}$ is larger than the value of the Rayleigh-Bénard problem. When the heating is inward $\left(\gamma_{e}<0\right)$, the instability occurs only in the second regime. The stabilization is more important for smaller $\eta$, requiring more work to the basic gravity for instability.

Figure 11a shows normalized $W_{\mathrm{BG}}$ and $D_{\mathrm{v}}$ for different azimuthal mode number $n$ for small $\eta$ in an outward heating. The Rayleigh number is fixed at the critical value $L_{c}$. The work done by the perturbation gravity is negligible $\left(W_{\mathrm{PG}} / 2 K \approx-0.05\right)$. As $n$ increases, the $W_{\mathrm{BG}}$ curve is first lowered and then shifts upward, meaning that the energy generation by the basic gravity becomes efficient beyond a certain $n(\neq 0)$. The viscous dissipation $D_{\mathrm{v}}$ exhibits similar behavior. The critical mode $(n=2, q=3.014)$ is a compromise from the competition of $W_{\mathrm{BG}}$ and $D_{\mathrm{v}}$. For large $\eta$, the work by the perturbation gravity plays an important role. Figure $11 \mathrm{~b}$ shows its stabilizing contribution $\left(-W_{\mathrm{PG}}\right)$ with $W_{\mathrm{BG}}$ and $D_{\mathrm{v}}$. The stabilization by $W_{\mathrm{PG}}$ is large for small $q$, making the critical modes' wavenumber larger. The $W_{\mathrm{PG}}$ contribution in an inward heating is also shown in the figure. The more significant contribution of $W_{\mathrm{PG}}$ results in $q_{c}$ larger than in the outward heating case (Fig. 8). 

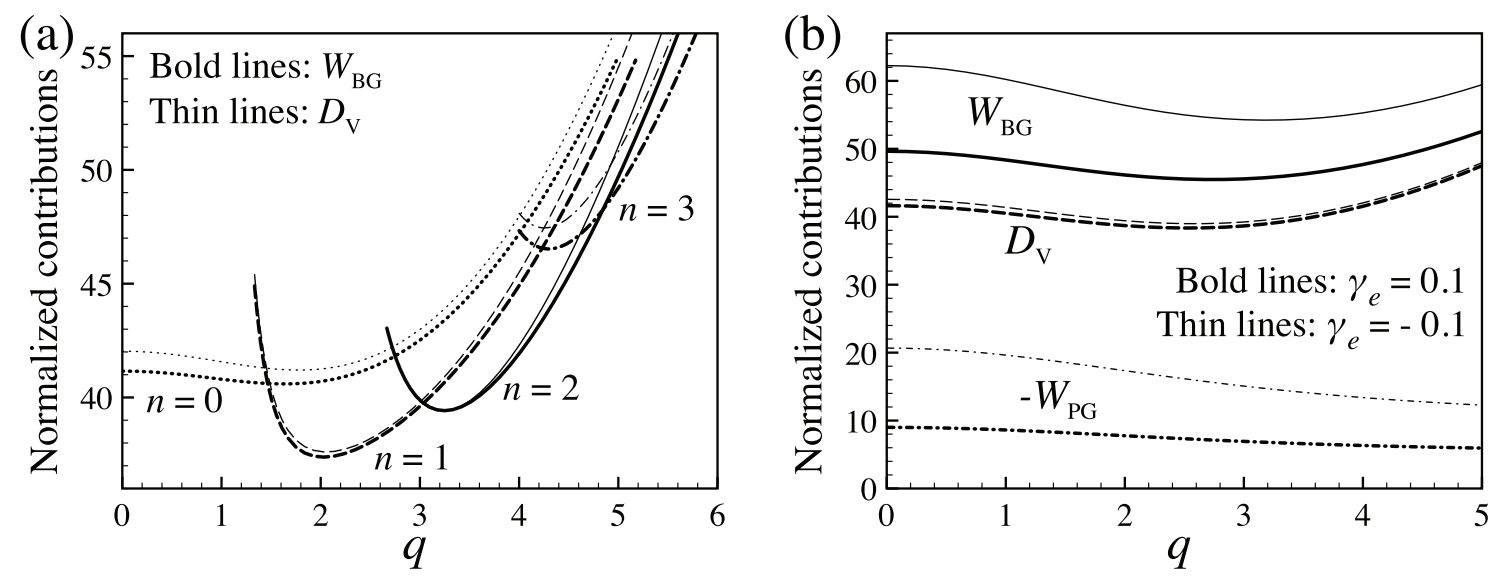

FIG. 11. Normalized different contributions to the rate of change of the convection flow kinetic energy (Eq. 29) at critical Rayleigh numbers: (a) at a small radius ratio $\eta$ for different azimuthal mode number $n$ in an outward heating $\left(\eta=0.2, \gamma_{e}=0.01\right.$ and $\left.L=888.4\right)$ and (b) at a large $\eta$ for different heating directions $\left(\eta=0.98 ; L=2005\right.$ and 2355 for $\gamma_{e}=0.1$ and -0.1 , respectively). The Prandtl number is fixed at 10 for all.

\section{DISCUSSION \& CONCLUSION}

The energy analysis in Sec. V showed that the basic electric gravity (15) provokes thermal instability against stabilizing effects of dissipations. The stability is hence well described by the Rayleigh number based on the basic gravity. In experiments, however, the main control parameters will be the electric potential $V_{0}$ and the temperature difference $\Delta \theta$. Figure 12 shows stability boundaries on the temperature-voltage plane for different $\eta$. The voltage is nondimensionalized by the reference voltage $V_{\text {ref }}$ defined by $V_{\text {ref }}=\left(\rho \kappa \nu / \epsilon_{2}\right)^{1 / 2}$. It takes a value, for example, $V_{\text {ref }}=6.2 \mathrm{~V}$ for the electrical grade silicone oil used by Chandra \& Smylie. ${ }^{7}$ For an outward heating $\left(\gamma_{e}>0\right)$, the basic state is more stable for larger $\eta$ and approaches the curve of the plane geometry $\operatorname{problem}^{9}\left(\gamma_{e}= \pm \sqrt{2129}\left(V_{0} / V_{\text {ref }}\right)^{-1}\right)$ in the narrow gap limit. For an inward heating $\left(\gamma_{e}<0\right)$, increase in $\eta$ destabilizes the system, indicating again the stability asymmetry with respect to the heating direction.

The theoretical results of Chandra \& Smylie $^{7}$ and those of Takashima ${ }^{8}$ are also presented in the diagram. These authors considered annuli with large $\eta$, where the mode degeneracy is important (Sec. IV A). Even though their analyses are restricted to axisymmetric disturbances, hence, the results will provide good predictions on the instability thresholds. Indeed, the theories agree well with the present one for small $\left|\gamma_{e}\right|$. The discrepancy found at large $\left|\gamma_{e}\right|$ is due to the complete and partial omission of the thermo-electro coupling: 


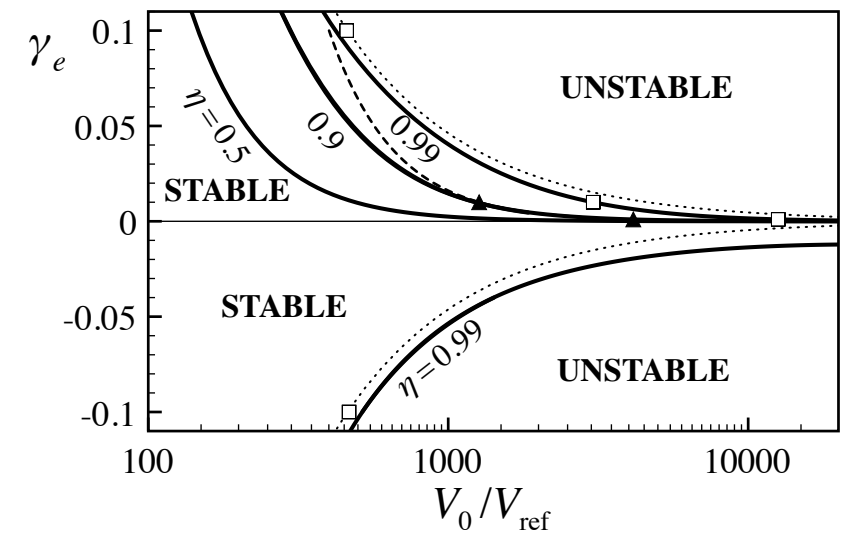

FIG. 12. Stability boundaries in the voltage-temperature plane for different radius ratio $\eta$. Theoretical predictions of the existing theoretical works are also shown (…….... Takashima \& Hamabata ${ }^{9}$ for plane geometry; - - - : Chandra \& Smylie $^{7}$ for $\eta=0.89 ; \boldsymbol{\Delta}$ and $\square$ : Takashima ${ }^{8}$ for $\eta=0.91$ and $\eta=0.99$, respectively).

Chandra \& Smylie neglected the $\gamma_{e}$-dependence of the basic electric gravity as well as the thermo-electro feedback in perturbations; Takashima's theory includes the coupling but only on the first order.

In the present paper, we considered the stability of a dielectric liquid layer in an annulus subjected to a radial temperature gradient and a radial high frequency electric field. The linear stability problem was formulated with regarding dielectrophoretic (DEP) effects as those due to the electric gravity. The predicted critical mode is made of stationary helices; this aspect is omitted in the previous theoretical work ${ }^{7,8}$ which concerns only axisymmetric perturbations. In the electric gravity, we distinguished its basic component $\bar{g}_{e}$ and perturbation component (25): the former is the analogue of the terrestrial gravity in the ordinary thermal convection problem, while the latter represents the feedback of electric field to temperature disturbances. A detail analysis from an energetic viewpoint showed that the basic electric gravity provokes the instability, while the perturbation gravity has a stabilizing effect. For the outward heating $\left(\gamma_{e}>0\right)$, its contribution is negligible at small radius ratio $\eta(\eta \lesssim 0.6)$. For large $\eta$, in contrast, it is responsible for the increase of $L_{c}$ as $\eta \rightarrow 1$ and explains why the critical Rayleigh number of the DEP thermal convection in the plane geometry is larger than that of the Rayleigh-Bénard problem. In the inward heating, the instability is observed only in narrow gaps $\gamma_{e}<\log \eta /(1+\log \eta)$. The critical Rayleigh number $L_{c}$ is larger than in the outward heating, as the stabilization by the perturbation gravity is more prominent. 
These results validate in the framework of linear theory the analogy with the gravity-driven thermal instability and clarify its limit.

\section{ACKNOWLEDGMENTS}

This work has been partly supported by the CNES (Centre National d'Etudes Spatiales) and the CNRS (Centre National de la Recherche Scientifique). H.N.Y. thanks the Université du Havre and the FEDER (Fonds Européen de Développement Régional) for his postdoctoral grant.

\section{Appendix A: Numerical method}

The unknown functions in Eqs. (18)-(23) are developed into a series of the Chebyshev polynomials $\left\{T_{l}(\xi)\right\}(l=0,1, \ldots, N)$ with $\xi=2 r-(1+\eta) /(1-\eta)$, e.g., $\tilde{u}=U_{0} T_{0}(\xi)+$ $U_{1} T_{1}(\xi)+\cdots+U_{N} T_{N}(\xi)$. The set of coefficients $\left\{U_{l}\right\}$ is the spectrum of $\tilde{u}$ in the Chebyshev space. We seek flows that satisfies Eqs. (18)-(23) inside the gap at the Chebyshev-GaussLobatto collocation points $\xi=\xi_{j}=\cos (j \pi / N)(j=1, \ldots, N-1)$ as well as the boundary conditions (27) on the cylinder surfaces.

In terms of the Chebyshev spectra, the governing equations (18)-(23) at the collocation points are written as follows:

$$
\begin{aligned}
& \left(2 \mathbf{K D}+\left[\frac{\mathrm{K}_{j l}}{r_{j}}\right]\right) \mathbf{U}+i n\left[\frac{\mathrm{K}_{j l}}{r_{j}}\right] \mathbf{V}+i k \mathbf{K W}=0, \\
& \left(\mathbf{L}-\left[\frac{\mathrm{K}_{j l}}{r_{j}^{2}}\right]\right) \mathbf{U}-2 i n\left[\frac{\mathrm{K}_{j l}}{r_{j}^{2}}\right] \mathbf{V}-2 \mathbf{K D \Pi}+L\left[\bar{g}_{e, j} \mathrm{~K}_{j l}\right] \boldsymbol{\Theta}-2 L C\left[\bar{\theta}_{j} \bar{E}_{j}^{\prime} \mathrm{K}_{j l}\right] \mathbf{D} \boldsymbol{\Phi} \\
& 2 i n\left[\frac{\mathrm{K}_{j l}}{r_{j}^{2}}\right] \mathbf{U}+\left(\mathbf{L}-\left[\frac{\mathrm{K}_{j l}}{r_{j}^{2}}\right]\right) \mathbf{V}-i n\left[\frac{\mathrm{K}_{j l}}{r_{j}}\right] \mathbf{\Pi}-2 i n L C\left[\frac{\left.\bar{\theta}_{j} \bar{E}_{j} \mathrm{~K}_{j l}\right] \mathbf{D}_{j} \mathrm{~K}_{j l} \mathbf{\Phi}=\operatorname{Pr}^{-1} s \mathbf{K U},}{r_{j}}\right] \mathbf{D \Phi}=\operatorname{Pr}^{-1} s \mathbf{K V}, \\
& \mathbf{L W}-i k \mathbf{K \Pi}-2 i k L C\left[\bar{E}_{j} \mathrm{~K}_{j l}\right] \mathbf{D} \boldsymbol{\Phi}=\operatorname{Pr}^{-1} s \mathbf{K W}, \\
& -\left[\bar{\theta}_{j}^{\prime} \mathrm{K}_{j l}\right] \mathbf{U}+\mathbf{L \Theta}=\operatorname{Pr}^{-1} s \mathbf{K \Theta}, \\
& -\gamma_{e}\left(2\left[\bar{\phi}_{j}^{\prime} \mathrm{K}_{j l}\right] \mathbf{D}+\left[\bar{\phi}_{j}^{\prime \prime} \mathrm{K}_{j l}\right]+\left[\frac{\bar{\phi}_{j}^{\prime} \mathrm{K}_{j l}}{r_{j}}\right]\right) \boldsymbol{\Theta}+\left[\bar{\epsilon}_{j} \mathrm{~L}_{j l}\right] \mathbf{\Phi}+2\left[\bar{\epsilon}_{j}^{\prime} \mathrm{K}_{j l}\right] \mathbf{D} \boldsymbol{\Phi}=0 .
\end{aligned}
$$

where $\mathbf{U}, \mathbf{V}, \mathbf{W}, \boldsymbol{\Pi}, \boldsymbol{\Theta}$ and $\boldsymbol{\Phi}$ are the column vectors consisting of the spectra of $\tilde{u}, \tilde{v}, \tilde{w}, \tilde{\pi}$, 
$\tilde{\theta}$ and $\tilde{\phi}$, respectively, e.g., $\mathbf{U}=\left[U_{0} U_{1} \ldots U_{N}\right]^{T}$. The square brackets signify a matrix such that the $(j, l)$ entry of $\left[A_{j l}\right]$ is $A_{j l}(j=1,2, \ldots, N-1 ; l=1,2, \cdots, N+1)$. The basic fields at $r=r_{j}$ are denoted like $\bar{g}_{e, j}=\bar{g}_{e}\left(r_{j}\right), \bar{\theta}_{j}=\bar{\theta}\left(r_{j}\right), \bar{\theta}_{j}^{\prime}=\bar{\theta}^{\prime}\left(r_{j}\right)$, and so on. The transformation matrix $\mathbf{K}=\left[\mathrm{K}_{j l}\right]$, the derivation matrix $\mathbf{D}=\left[\mathrm{D}_{l m}\right]$ and the Laplacian matrix $\mathbf{L}=\left[\mathrm{L}_{j l}\right]$ are defined as:

$$
\begin{gathered}
\mathrm{K}_{j l}=\cos \frac{j l \pi}{N}, \quad \mathrm{D}_{l m}=\left\{\begin{array}{l}
m-1 \quad(l=1 ; m=2,4, \cdots), \\
2(m-1)(l \geqq 2 ; m=l+1, l+3, \cdots), \\
0 \quad \text { (otherwise) },
\end{array}\right. \\
\mathbf{L}=4 \mathbf{K D}^{2}+2\left[\frac{\mathrm{K}_{j l}}{r_{j}}\right] \mathbf{D}-\left[\frac{\mathrm{K}_{j l}}{r_{j}^{2}}\right]-k^{2} \mathbf{K}
\end{gathered}
$$

for $j=1,2, \ldots, N-1$ and $l, m=1,2, \cdots, N+1$.

The boundary conditions (27) are written as

$$
\mathbf{H U}=\mathbf{H V}=\mathbf{H W}=\mathbf{H D U}=\mathbf{H \Theta}=\mathbf{H} \Phi=0
$$

where $\mathbf{H}=\left[\mathrm{H}_{j l}\right]$ is a $2 \times(N+1)$ matrix: $\mathrm{H}_{1 l}=1, \mathrm{H}_{2 l}=(-1)^{l-1}(l=1,2, \cdots, N+1)$. Its products with Chebyshev spectra give the values of functions on the inner and outer cylinders.

The $6(N+1)$ equations of $(\mathrm{A} 1)-(\mathrm{A} 6)$ and $(\mathrm{A} 9)$ with respect to $6(N+1)$ unknowns of $\mathbf{U}$, $\mathbf{V}, \mathbf{W}, \boldsymbol{\Pi}, \boldsymbol{\Theta}$ and $\boldsymbol{\Phi}$ define a generalized eigenvalue problem:

$$
\mathbf{A}\left(\eta, \gamma_{e}, L, n, k\right) \mathbf{X}=s \mathbf{B}(\operatorname{Pr}) \mathbf{X}
$$

where $\mathbf{X}=\left[\mathbf{U}^{T} \mathbf{V}^{T} \mathbf{W}^{T} \boldsymbol{\Pi}^{T} \mathbf{\Theta}^{T} \boldsymbol{\Phi}^{T}\right]^{T}$ and $\mathbf{A}$ and $\mathbf{B}$ are coefficient matrices. The eigenvalues and eigenvectors are computed by employing the $Q Z$-decomposition. The highest order of considered Chebyshev polynomials is typically set at $N=60$ to ensure the convergence.

\section{REFERENCES}

${ }^{1}$ R. Kronig and G. Ahsmann, "The influence of an electric field on the convective heat transfer in liquids," Appl. Sci. Res. Sect. A 2, 235-244 (1949). 
${ }^{2}$ T. B. Jones, "Electrohydrodynamically enhanced heat transfer in liquids - A review," Advances in Heat Transfer 14, 107-148 (1979).

${ }^{3}$ L. D. Landau and E. M. Lifshitz, Electrodynamics of Continuous Media, 2nd ed., Landau and Lifshitz Course of Theoretical Physics, Vol. 8 (Elsevier Butterworth-Heinemann, Burlington, Massachusetts, 1984).

${ }^{4}$ R. J. Turnbull, "Electroconvective instability with a stabilizing temperature gradient. I. Theory," Phys. Fluids 11, 2588-2596 (1968).

${ }^{5}$ R. J. Turnbull, "Electroconvective instability with a stabilizing temperature gradient. II. Experimental results," Phys. Fluids 11, 2597-2603 (1968).

${ }^{6}$ I. M. Yavorskaya, N. I. Fomina, and Y. N. Belyaev, "A simulation of central-symmetry convection in microgravity conditions," Acta Astronautica 11, 179-183 (1984).

${ }^{7}$ B. Chandra and D. E. Smylie, "A laboratory model of thermal convection under a central force field," Geophysical Fluid Dynamics 3, 211-224 (1972).

${ }^{8} \mathrm{M}$. Takashima, "Electrohydrodynamic instability in a dielectric fluid between two coaxial cylinders," Q. J. Mech. Appl. Math. 33, 93-103 (1980).

${ }^{9}$ M. Takashima and H. Hamabata, "The stability of natural convection in a vertical layer of dielectric fluid in the presence of a horizontal ac electric field," J. Phys. Soc. Jpn 53, 1728-1736 (1984).

${ }^{10}$ B. Sitte and H. J. Rath, "Influence of the dielectrophoretic force on thermal convection," Exp. Fluids 34, 24-27 (2003).

${ }^{11}$ R. J. Turnbull, "Effect of dielectrophoretic forces on the Bénard instability," Phys. Fluids 12, 1809-1815 (1969).

${ }^{12}$ P. J. Stiles, "Electro-thermal convection in dielectric liquids," Chem. Phys. Lett. 179, 311-315 (1991).

${ }^{13}$ P. J. Stiles, F. Lin, and P. J. Blennerhassett, "Convective heat transfer through polarized dielectric liquids," Phys. Fluids A 5, 3273-3279 (1993).

${ }^{14}$ V. Travnikov, C. Egbers, and R. Hollerbach, "The geoflow-experiment on ISS (Part II): Numerical simulation," Adv. Space Res. 32, 181-189 (2003).

${ }^{15}$ B. Futterer, R. Hollerbach, and C. Egbers, "GeoFlow: 3D numerical simulation of supercritical thermal convective states," J. Phys.: Conf. Ser. 137, 012026 (2008).

${ }^{16}$ F. Feudel, K. Bergemann, L. S. Tuckerman, C. Egbers, B. Futterer, and R. Hollerbach, "Convection patterns in a spherical fluid shell," Phys. Rev. E 83, 046304 (2011). 
${ }^{17}$ B. Futterer, C. Egbers, N. Dahley, S. Koch, and L. Jehring, "First identification of suband supercritical convection patterns from 'GeoFlow', the geophysical flow simulation experiment integrated in Fluid Science Laboratory," Acta Astronautica 66, 193-200 (2010).

${ }^{18}$ B. Futterer, N. Dahley, S. Koch, N. Scurtu, and C. Egbers, "From viscous convective experiment 'GeoFlow I' to temperature-dependent viscosity in 'GeoFlow II' - Fluid physics experiments on-board ISS for the capture of convection phenomena in Earth's outer core and mantle," Acta Astronautica 71, 11-19 (2012).

${ }^{19}$ P. J. Stiles and M. Kagan, "Stability of cylindrical Couette flow of a radially polarised dielectric liquid in a radial temperature gradient," Physica A 197, 583-592 (1993).

${ }^{20}$ S. V. Malik, H. N. Yoshikawa, O. Crumeyrolle, and I. Mutabazi, "Thermo-electrohydrodynamic instabilities in a dielectric liquid under microgravity," Acta Astronautica 81, 563-569 (2012).

${ }^{21}$ R. J. Turnbull and J. R. Melcher, "Electrohydrodynamic Rayleigh-Taylor bulk instability," Phys. Fluids 12, 1160-1166 (1969).

${ }^{22}$ S. Chandrasekhar, Hydrodynamic and hydromagnetic stability (Dover, New York, 1961).

${ }^{23}$ M. Ali and P. D. Weidman, "On the stability of circular Couette flow with radial heating," J. Fluid Mech. 220, 53-84 (1990).

${ }^{24}$ R. Tagg and P. D. Weidman, "Linear stability of radially-heated circular Couette flow with simulated radial gravity," Z. Angew. Math. Phys. 58, 431-456 (2007). 